\title{
Epithelial-Mesenchymal Transition in a Case of Metastatic Thyroid Carcinoma in a Brown Bear (Ursus arctos)
}

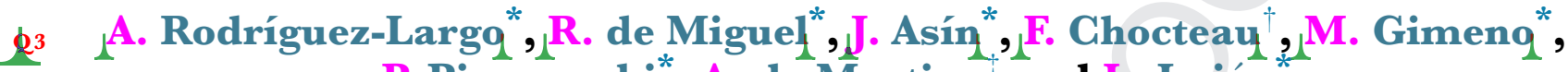

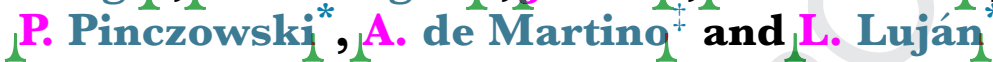 \\ * Department of Animal Pathology, University of Zaragoza, Zaragoza, Spain, ${ }^{\dagger}$ Laboniris, ONIRIS, Ecole Nationale \\ Vétérinaire, Agroalimentaire et de l'Alimentation de Nantes-Atlantique, Nantes, France and ${ }^{\ddagger}$ Instituto Aragonés de Ciencias de \\ la Salud, Zaragoza, Spain
}

\section{Summary}

A 20-year-old male brown bear (Ursus arctos) with a $20 \times 25 \mathrm{~cm}$ necrotic mass adjacent to the trachea was diagnosed as having an anaplastic thyroid carcinoma. Metastases were observed in the lungs and one adrenal gland and, histologically, these had anaplastic and follicular carcinoma patterns, respectively. E-cadherin labelling was observed in the adrenal mass only, while N-cadherin immunolabelling was detected in the thyroid gland and lung masses. Thyroid-specific markers (thyroid transcription factor-1, thyroglobulin) were expressed in the adrenal gland metastasis. This case illustrates an example of a primary epithelial-mesenchymal transition enabling metastasis to distant organ sites, followed by a mesenchymal-epithelial transition within the adrenal gland microenvironment, allowing invasion and reacquisition of thyroid epithelial cell features. Epithelial-mesenchymal transitions help to understand the phenomenon of carcinoma cell plasticity in enabling colonization and growth of metastases.

(C) 2020 Elsevier Ltd. All rights reserved.

Keywords: brown bear; epithelial-mesenchymal transition; thyroid carcinoma

Anaplastic thyroid carcinomas are one of the most aggressive solid tumours in dogs (Liptak, 2007) and man, but they represent only $1-2 \%$ of all thyroid neoplasms (Molinaro et al., 2017). Thyroid neoplasms tend to invade the bloodstream and have high rates of metastasis (Liptak, 2007). Thyroid gland carcinomas have been described in domestic and wild animals (Rosol and Gröne, 2016). A few cases of endocrine neoplasms have been described in the Ursidae family (Lombard and Witte, 1959; Hubbard et al., 1983).

Immunohistochemical characterization of thyroid neoplasms includes use of markers such as thyroid transcription factor (TTF)-1, a thyroid-specific protein, the expression of which confirms a thyroid origin

Correspondence to: L. Luján (e-mail: Lluis.Lujan@unizar.es).
(Ramos-Vara et al., 2016); thyroglobulin (TGB), a thyroid hormone used to classify thyroid neoplasms (Ramos-Vara et al., 2016); and cytokeratin and Ecadherin (Fadda and Rossi, 2014). E-cadherin is an adhesion protein with an important role in the formation of epithelial cell-to-cell junctions (Onder et al., 2008). Downregulation of E-cadherin is considered the crucial event in dedifferentiation, progression and metastasis of carcinomas (Liu and Lin, 2015). Mesenchymal markers such as vimentin and N-cadherin can be used to complement the information provided by these antibodies (Onder et al., 2008).

Epithelial-mesenchymal transition (EMT) is a cellular process characterized by the conversion of epithelial cells to a mesenchymal phenotype. EMT plays a physiological role during embryogenesis (Lee et al., 2005). This process is silenced during 
adulthood, but it can be reactivated by different pathological conditions (Zidar et al., 2018; Dongre and Weinberg, 2019). EMT allows primary neoplastic epithelial cells to acquire the capacity to migrate and to metastasize (Lee et al., 2005). In these newlycolonized tissues, neoplastic cells can undergo the so-called mesenchymal-epithelial transition (MET), a reverse process that can occur in response to different microenvironmental signals. As a consequence, metastases can re-acquire a differentiated epithelial phenotype similar to that of the normal tissue where the metastatic tumour first appeared (Thiery, 2002).

Herein we report the degrees of cellular differentiation in pulmonary and adrenal gland metastases observed in a case of anaplastic thyroid carcinoma in a brown bear (Ursus arctos). These findings and the immunohistochemical features, suggested an EMT process in the thyroid neoplastic cells and a MET process in the adrenal gland metastases.

A 20-year-old male brown bear, born and raised at a circus, was referred with an expanding, firm mass adjacent to the trachea. Dyspnoea, anorexia and progressive weight loss were the major clinical signs. Radiographical examination of the lungs revealed multiple pulmonary nodules and the animal was humanely destroyed.

At necropsy examination, an irregular, firm dark brown, solid mass $(20 \times 25 \mathrm{~cm})$ was found in the thyroid region. The cut surface revealed a large central necrotic area (approximately $22 \mathrm{~cm}$ in diameter), with a $2 \mathrm{~cm}$ ring of peripheral viable tissue. The lungs exhibited multiple nodules of different sizes $(0.5-15 \mathrm{~cm})$, distributed randomly and associated with an intense mediastinal proliferation (Supplementary Fig. 1). In the right adrenal gland, a pale yellow mass $(3 \times 5 \mathrm{~cm})$ mass was present (Supplementary Fig. 2). Tissue samples were processed routinely and embedded in paraffin wax. Sections were stained by haematoxylin and eosin (HE) and serial sections were subjected to immunohistochemistry (IHC) (Supplementary Table 1).

Microscopically, the thyroid mass was encapsulated, highly cellular and had a broad necrotic centre. The neoplasm was composed of polyhedral to spindleshaped cells either in sheets or in interlacing bundles, streams and whorls. The cells were ill-defined, and showed a variable amount eosinophilic cytoplasm (Supplementary Fig. 3). Nuclei varied in size and shape and had one to four prominent eosinophilic nucleoli (Supplementary Fig. 4). Anisokaryosis and anisocytosis were marked and the mitotic index was high $(11$ mitoses per $10 \times 400$ fields). No normal thyroid tissue was observed. Aggregates of neoplastic cells were frequently observed in an intravascular location
(Fig. 1). Pulmonary nodules showed a histological pattern similar to the thyroid mass, without identifiable normal thyroid tissue (Supplementary Figs. 5 and 6). The adrenal gland metastasis was organized in variably-sized follicles with intraluminal eosinophilic homogeneous material, resembling colloid (thyroid follicle-like structures), sometimes surrounded by solid areas of polygonal, thyroid-like neoplastic cells. Anisokaryosis and anisocytosis were mild and the mitotic index was low (1 mitosis per $10 \times 400$ fields) (Supplementary Figs. 7 and 8). Neoplastic cells in the thyroid and lung masses were negative for E-cadherin, but had cytoplasmic expression of N-cadherin (Figs. 1 and 2; Supplementary Figs. 9 and 10). Moreover, the neoplastic cells exhibited strong cytoplasmic expression of vimentin. In contrast, cells in the adrenal mass showed the reverse pattern of immunolabelling, having strong cytoplasmic reactivity for E-cadherin, low signal for vimentin and no N-cadherin expression (Figs. 3 and 4, Supplementary Table 2). The cytoplasm of the neoplastic cells in the thyroid, lung and adrenal tumour masses were strongly positive for pancytokeratin (Supplementary Figs. 11,13 and 15). TTF-1 and TGB were expressed sparsely in the thyroid and lung masses, but the adrenal metastases had low nuclear expression of TTF-1 and strong cytoplasmic reactivity against TGB (Supplementary Figs. 12,14 and 16).

Based on these findings, an anaplastic thyroid carcinoma with metastases to the lung and the adrenal gland was diagnosed. Lung metastases showed an undifferentiated, anaplastic growth pattern, similar to the proliferation observed in the thyroid gland. However, the adrenal mass exhibited well-differentiated cell proliferation with recognizable thyroid follicle-

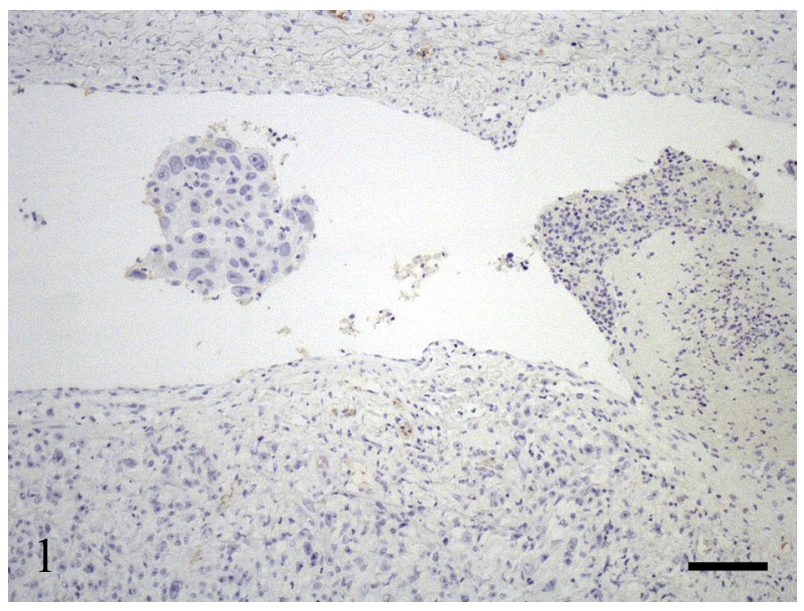

Fig. 1. Thyroid. Cluster of neoplastic cells within a blood vessel are negative for E-cadherin. IHC. Bar, $100 \mu \mathrm{m}$. 


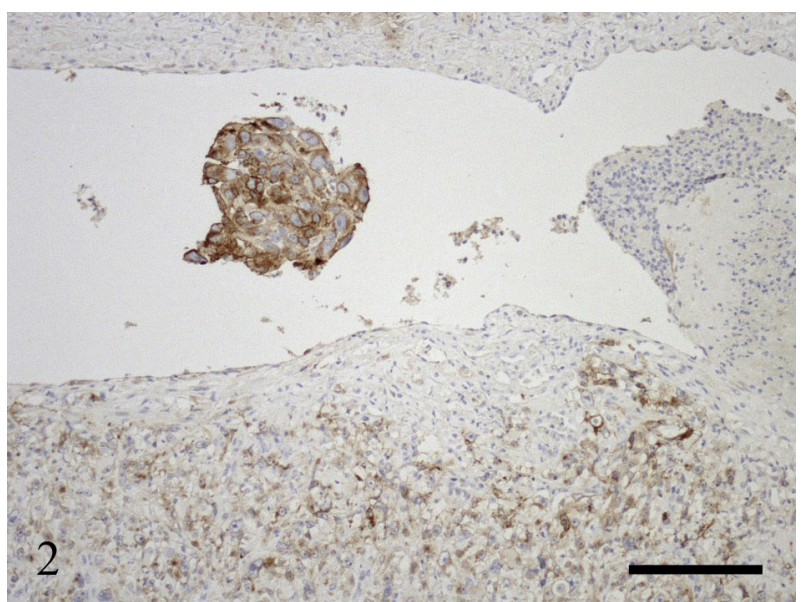

Fig. 2. Thyroid. Anaplastic cells are diffusely positive for N-cadherin. IHC. Bar, $100 \mu \mathrm{m}$.

like structures and the tumour was eventually diagnosed as a metastatic follicular thyroid carcinoma.

IHC using a variety of antibodies demonstrated a thyroid-derived epithelial tumour. The primary and pulmonary masses were positive for mesenchymal markers and negative for the most common thyroid markers (TTF-1 and TGB), indicating a high level of anaplasia. However, the metastatic adrenal mass retained positivity for these two markers and also for E-cadherin, indicating a more differentiated neoplasm. Multiple endocrine neoplasia is characterized by the appearance of different primary tumours within two or more endocrine glands at the same time (Roccabianca et al., 2006), a different scenario to the present case.

Ectopic thyroid tissue is normally found in areas such as the anterior tongue, trachea and larynx and such tis-

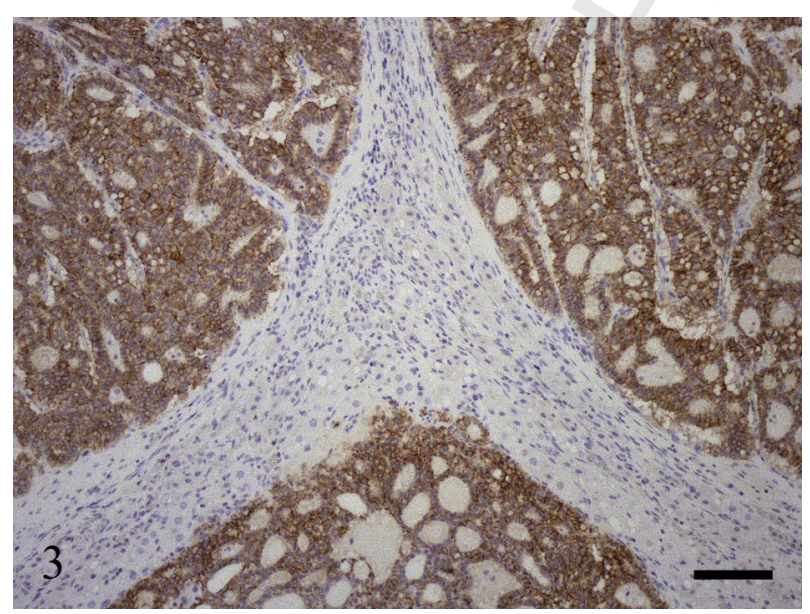

Fig. 3. Thyroid carcinoma, metastasis in adrenal. Neoplastic cells are diffusely positive for E-cadherin, stroma is negative. IHC. Bar, $100 \mu \mathrm{m}$.

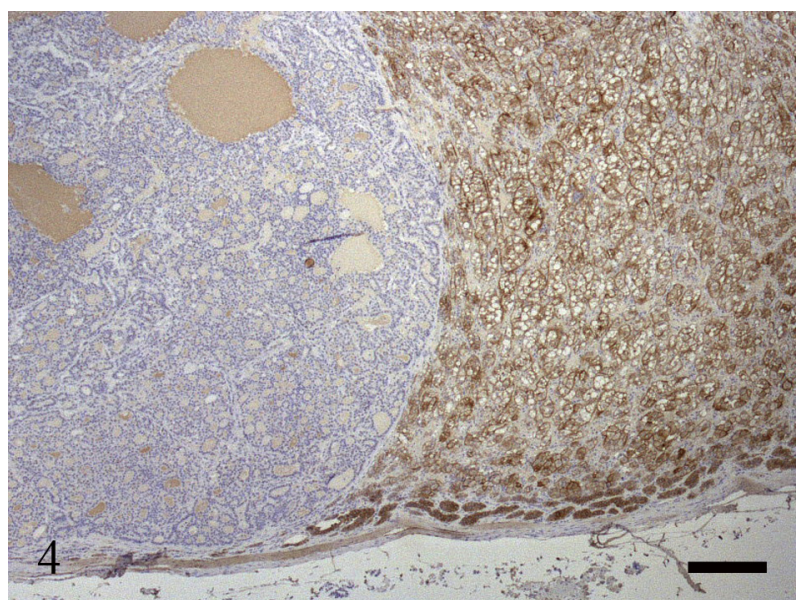

Fig. 4. Thyroid carcinoma, metastasis in adrenal. Neoplastic cells are negative for $\mathrm{N}$-cadherin. Normal adrenal cortex cells exhibit strong labelling. IHC. Bar, $250 \mu \mathrm{m}$.

sue might appear in the adrenal gland (Shuno et al., 2006). Remarkably, metastasis from thyroid carcinomas occasionally exhibit a better-differentiated appearance than the primary tumour, sometimes leading to misdiagnosis of ectopic thyroid tissue rather than neoplasia (Kondo et al., 2000; Shuno et al., 2006).

This case illustrates an example of tumour cell plasticity: a primary EMT in the thyroid gland that enabled the metastatic process, followed by a MET that allowed the neoplastic cells to adapt to a new microenvironment and to re-acquire their differentiated epithelial cell features in the adrenal gland. Metastasis is a highly inefficient multistep process, only a small number of the circulating neoplastic cells are able to survive and thrive in a foreign microenvironment (Tsai and Yang, 2013). The initial metastatic events of carcinomas frequently depend on EMT activation, a partial transition of the epithelial neoplastic cells into a mesenchymal phenotype that allows loss of cell-to-cell adhesion, disaggregation and enhancement of their motility and invasive properties (Kalluri and Weinberg, 2009). In the present case, EMT was demonstrated in the primary thyroid mass and in the lung metastases by the lowered expression of $\mathrm{E}$-cadherin and the increased expression of $\mathrm{N}$-cadherin and vimentin, together with the acquisition of a spindle-shaped mesenchymal morphology by the neoplastic cells (Knaack et al., 2018). In contrast, MET was demonstrated in the adrenal metastases by the re-expression of E-cadherin and the loss of N-cadherin and vimentin signals, together with the re-establishment of an epithelial cell morphology. These features allowed the recognition of the primary tissue from which the tumour arose, something that was not feasible in the metastases in the lungs. The interactions between invading 
neoplastic cells and the new microenvironment can modulate their outgrowth, progression and final cell morphology and phenotype (Gao et al., 2012). EMT has been demonstrated in different neoplasms including mammary or pancreatic carcinomas (Dongre and Weinberg, 2019). The present case shows a common adaptive process in carcinomas that possibly occurs in all species, and may explain the tumour cell plasticity which enables colonization and growth of neoplasia in distant organ sites.

\section{Acknowledgments}

The current address of J. Asin is California Animal Health \& Food Safety Laboratory System, Pathology, Microbiology \& Immunology Department, University of California at Davis, Davis, California, USA. The current address of M. Gimeno is University Veterinary Teaching Hospital Camden, The University of Sydney, Australia. The current address of P. Pinczowski is NSW Department of Primary Industries/ Biosecurity \& Food Safety, EMAI, Menangle, Australia.

\section{Supplementary data}

Supplementary data to this article can be found online at https://doi.org/10.1016/j.jcpa.2020.01.004.

\section{Uncited Reference}

Rosol and Meuten, 2017.

\section{References}

Dongre A, Weinberg RA (2019) New insights into the mechanisms of epithelial- mesenchymal transition and implications for cancer. Nature Reviewes Molecular Cell Biology, 20, 69-84.

Fadda G, Rossi ED (2014) Immunohistochemical diagnosis of thyroid tumors. Surgical Pathology Clinics, 7, 491-500.

Gao D, Vahdat LT, Wong S, Chang JC, Mittal V (2012) Microenvironmental regulation of epithelialmesenchymal transitions in cancer. Cancer Research, 72, 4883-4889.

Hubbard GB, Schmidt RE, Fletcher KC (1983) Neoplasia in zoo animals. Fournal of Zoo Animal Medicine, 14, $33-40$.

Kalluri R, Weinberg RA (2009) The basics of epithelial-mesenchymal transition. Fournal of Clinical Investigation, 119, 1420-1428.

Knaack H, Lenk L, Philipp L-M, Miarka L, Rahn S et al. (2018) Liver metastasis of pancreatic cancer: the hepatic microenvironment impacts differentiation and selfrenewal capacity of pancreatic ductal epithelial cells. Oncotarget, 9, 31771-31786.
Kondo T, Katoh R, Omata K, Oyama T, Yagawa A et al. (2000) Incidentally detected liver metastasis of welldifferentiated follicular carcinoma of the thyroid, mimicking ectopic thyroid. Pathology International, 50, 509-513.

Lee JM, Dedhar S, Kalluri R, Thompson EW (2005) The epithelial-mesenchymal transition: new insights in signaling, development, and disease. Fournal of Cell Biology, 172, 973-981.

Liptak JM (2007) Canine thyroid carcinoma. Clinical Techniques in Small Animal Practice, 22, 75-81.

Liu H, Lin F (2015) Application of immunohistochemistry in thyroid pathology. Archives of Pathology and Laboratory Medicine, 139, 67-82.

Lombard IS, Witte EJ (1959) Frequency and types of tumors in mammals and birds of the Philadelphia Zoological Garden. Cancer Research, 19, 127-141.

Molinaro E, Romei C, Biagini A, Sabini E, Agate L et al. (2017) Anaplastic thyroid carcinoma: from clinicopathology to genetics and advanced therapies. Nature Reviewes Endocrinology, 13, 644-660.

Onder TT, Gupta PB, Mani SA, Yang J, Lander ES et al. (2008) Loss of E-cadherin promotes metastasis via multiple downstream transcriptional pathways. Cancer Research, 68, 3645-3654.

Ramos-Vara JA, Frank CB, DuSold D, Miller MA (2016) Immunohistochemical detection of Pax8 and Napsin A in canine thyroid tumours: comparison with thyroglobulin, calcitonin and thyroid transcription factor 1. Fournal of Comparative Pathology, 155, 286-298.

Roccabianca P, Rondena M, Paltrinieri S, Pocacqua V, Scarpa P et al. (2006) Multiple endocrine neoplasia type-I-like syndrome in two cats. Veterinary Pathology, 43, 345-352.

Rosol TJ, Gröne A (2016) Endocrine glands. In: Palmer's Pathology of Domestic Animals, $6^{\text {th }}$ Edit., MG Maxie, Kennedy Jubb, Ed., Elsevier, St. Louis, pp. 326-332.

Rosol T, Meuten D (2017) Tumors of the endocrine glands. In: Tumors of Domestic Animals, $5^{\text {th }}$ edit., DJ Meuten, Ed., John Wiley \& Sons, Ames, pp. 797-799.

Shuno Y, Kobayashi T, Morita K, Shimizu S, Nishio Y et al. (2006) Ectopic thyroid in the adrenal gland presenting as cystic lesion. Surgery, 139, 580-582.

Thiery JP (2002) Epithelial-mesenchymal transitions in tumour progression. Nature Reviews Cancer, 2, 442-454.

Tsai JH, Yang J (2013) Epithelial-mesenchymal plasticity in carcinoma metastasis. Genes \& Development, 27, 2192-2206.

Zidar N, Boštjančič E, Malgaj M, Gale N, Dovšak T et al. (2018) The role of epithelial-mesencymal transition in squamous cell carcinoma of the oral cavity. Virchow's Archive, 472, 237-245. 